MITSUBISHI ELECTRIC RESEARCH LABORATORIES

https://www.merl.com

\title{
Constrained Spacecraft Attitude Control on SO(3) Using Reference Governors and Nonlinear Model Predictive Control
}

\author{
Kalabić, U.; Gupta, R.; Di Cairano, S.; Bloch, A.; Kolmanovsky, I. \\ TR2014-047 June 04, 2014
}

\begin{abstract}
We develop nonlinear reference governor and nonlinear model predictive control schemes for constrained spacecraft attitude control. The schemes use the nonlinear discrete-time model of spacecraft dynamics based on the Lie group variational integrator evolving on $\mathrm{SO}(3) \mathrm{x}$ $\mathrm{SO}(3)$. The reference governor is a computationally simpler add-on to the nominal controller, while the model predictive controller provides faster response and better performance. The stability properties and constrained domains of attraction of these schemes are analyzed and their capability to perform global rest-to-rest reorientation maneuvers is established. Simulation results are reported where controllers perform specified reorientation maneuvers while adhering to torque and inclusion zone constraints.
\end{abstract}

American Control Conference (ACC)

(C) 2014 MERL. This work may not be copied or reproduced in whole or in part for any commercial purpose. Permission to copy in whole or in part without payment of fee is granted for nonprofit educational and research purposes provided that all such whole or partial copies include the following: a notice that such copying is by permission of Mitsubishi Electric Research Laboratories, Inc.; an acknowledgment of the authors and individual contributions to the work; and all applicable portions of the copyright notice. Copying, reproduction, or republishing for any other purpose shall require a license with payment of fee to Mitsubishi Electric Research Laboratories, Inc. All rights reserved. 



\title{
Constrained Spacecraft Attitude Control on $\mathrm{SO}(3)$ Using Reference Governors and Nonlinear Model Predictive Control
}

\author{
Uroš Kalabić \\ Rohit Gupta \\ Stefano Di Cairano \\ Anthony Bloch \\ Ilya Kolmanovsky
}

\begin{abstract}
Nonlinear reference governor and nonlinear model predictive control schemes are developed for constrained spacecraft attitude control. The schemes use the nonlinear discrete-time model of spacecraft dynamics based on the Lie group variational integrator evolving on $\mathrm{SO}(3) \times \mathrm{SO}(3)$. The reference governor is a computationally simpler add-on to the nominal controller, while the model predictive controller provides faster response and better performance. The stability properties and constrained domains of attraction of these schemes are analyzed and their capability to perform global rest-to-rest reorientation maneuvers is established. Simulation results are reported that demonstrate the capabilities of the controllers to perform specified reorientation maneuvers while adhering to torque and inclusion zone constraints.
\end{abstract}

\section{INTRODUCTION}

In this paper, we consider two schemes for constrained control of spacecraft maneuvers that exploit predictions based on discrete-time models with dynamics that evolve on $\mathrm{SO}(3) \times \mathrm{SO}(3)$. The first scheme that we consider is the reference governor, which makes online predictions in order to enforce pointwise-in-time state and control constraints through the choice of an admissible reference input. The theory of nonlinear reference governors [1], [2] has been developed for dynamics and constraints in $\mathbb{R}^{n}$, and here we extend the treatment to the case of $\mathrm{SO}(3) \times \mathrm{SO}(3)$. The second scheme employs model predictive control [3]-[6] designed for dynamics evolving on $\mathrm{SO}(3) \times \mathrm{SO}(3)$. Model predictive control solves a finite-time state- and controlconstrained control problem that guarantees asymptotic stability of the desired equilibrium. Compared to the reference governor, the model predictive controller provides better performance at the cost of increased computing effort.

Model predictive control approaches to constrained spacecraft attitude control have been considered in [7]-[9] based on linearized models. In this paper we pursue nonlinear reference governor and model predictive control approaches that are based on spacecraft nonlinear dynamics on $\mathrm{SO}(3) \times$ $\mathrm{SO}(3)$ and obtain global convergence properties.

Although other nonlinear methods of predictive control have been considered for dynamics partially evolving on

This work was supported in part by National Science Foundation Grants 1130160, DMS-0907949, 1207693, and Inspire-1343720.

U. Kalabić, R. Gupta, and I. Kolmanovsky are with the Department of Aerospace Engineering, University of Michigan, Ann Arbor, MI 48109 $\{$ kalabic, rohitgpt, ilya\}@umich.edu

S. Di Cairano is with Mitsubishi Electric Research Laboratories, Mechatronics, Cambridge, MA 02139 dicairano@ieee.org

This work was not sponsored by Mitsubishi Electric Co. or any of its subsidiaries.

A. Bloch is with the Department of Mathematics, University of Michigan, Ann Arbor, MI 48109 abloch@umich. edu
$\mathrm{SO}(3)$ [10], all of them calculate their predicted values through numerical integration methods that do not preserve the conserved quantities of motion, such as momentum and energy, and this can lead to large differences between the true and predicted values [11]. In contrast to previous methods, the reference governor and model predictive control schemes presented in this work utilize a Lie group variational integrator [11]-[17] as a predictive model for the rotational dynamics of a spacecraft.

Lie group variational integrators have been developed to exploit the underlying group structure and have been shown to preserve the conserved quantities of motion to machine precision. Standard integration schemes such as RungeKutta fail to achieve this, because they do not exploit the group structure. The Lie group variational integrator updates the rotation matrix by multiplying two matrices in $\mathrm{SO}(3)$, thereby ensuring that the rotation matrix evolves on $\mathrm{SO}(3)$ and the conserved quantities of motion are preserved. For other types of variational integrators, see [18], [19].

Theoretical results presented in this paper show recursive feasibility and convergence for both predictive control schemes; furthermore, in both cases, we show that the schemes have global convergence properties for all states satisfying initial feasibility with respect to constraints - this amounts to global stabilization if there are no constraints.

Simulations illustrating the methods above are also reported. We simulate rest-to-rest maneuvers of spacecraft attitude control, where the objective of the spacecraft attitude control problem is to command the spacecraft from a given attitude to the equilibrium in the non-inertial frame. The controller that we propose exploits the fact that model predictive control is able to generate discontinuous feedback laws, as shown in [20]. Additionally, in the case of model predictive control, we also command the spacecraft to a $180^{\circ}$ rotational rest-to-rest maneuver; this maneuver is notable because a discontinuity occurs at the $180^{\circ}$ initial rotation as a consequence of the Lefschetz-Hopf Theorem [21], which states that the sum of the indices of fixed points of a map on a manifold must be equal to the Lefschetz number of the map. In the case where the map is homotopic to the identity, the Lefschetz number is equal to the Euler characteristic of the manifold. As described above, the discrete state-update equation that we use in this paper is obtained as an approximation to a continuous-time system with no discontinuities, so it is homotopic to the identity. The Euler characteristic $\chi(\mathrm{SO}(3) \times \mathrm{SO}(3))=0$, but the index of a sink is 1 , implying that any globally stabilizing control on $\mathrm{SO}(3)$ is discontinuous. 
The paper is organized as follows. The remainder of this section describes the notation. In Section II, we describe the Lie group variational integrator that we employ for making predictions on $\mathrm{SO}(3) \times \mathrm{SO}(3)$. In Section III, we introduce the reference governor. In Section IV, we describe the model predictive control scheme and establish its recursive feasibility and stability properties. In Section V, we present numerical results for the two predictive control schemes obtained by simulating rotational maneuvers. Section VI is the conclusion.

\section{A. Notation}

The notation is mostly familiar with a few notable exceptions. $\mathbb{Z}_{N}$ denotes the set of the first $N$ nonnegative integers, $0,1, \ldots, N-1$, and $\mathbb{Z}_{+}$denotes the set of all nonnegative integers. Positive semi-definite and orthogonal matrices are written in uppercase bold. Elements of $\mathbb{R}^{3}$ are written in capital Greek letters. Given $A \in \mathbb{R}^{3}, A^{\times}$ denotes the corresponding value in the special orthogonal Lie algebra $\mathfrak{s o}(3)$, so that if $\mathrm{A}=\sum_{i=1}^{3} \lambda_{i} e_{i}$, where $e_{i}$ are the standard basis elements of $\mathbb{R}^{3}$, then $\mathrm{A}^{\times}=\sum_{i=1}^{3} \lambda_{i} j_{i}$, where $j_{i}$ are the standard basis elements of $\mathfrak{s o}(3) . \mathbf{I} \in \mathbb{R}^{3 \times 3}$ is the identity matrix. For a matrix $A \in \mathbb{R}^{3 \times 3}$, its trace is given by $\operatorname{tr}(A)=\sum_{i} A_{i i}$. For a vector $a \in \mathbb{R}^{n}$, and a symmetric positive definite matrix $\mathbf{M} \in \mathbb{R}^{n \times n}$, we denote the square of the norm by $\|a\|_{\mathbf{M}}^{2}=a^{\mathrm{T}} \mathbf{M} a$. For a matrix $A \in \mathbb{R}^{n \times m}$, we denote the square of the Frobenius norm by $\|A\|_{F}^{2}=\sum_{i, j} A_{i j}^{2}$. For a set $\mathcal{A} \subset \mathcal{B}$, the interior with respect to $\mathcal{B}$ is given by $\operatorname{int}(\mathcal{A})$. Finally, for a sequence $\left\{v_{k}, v_{k+1}, \ldots, v_{k+N}\right\}$, its predicted value at time-instant $k$ is denoted by $\left\{v_{k \mid k}, v_{k+1 \mid k}, \ldots, v_{k+N \mid k}\right\}$; note that $v_{k \mid k}=v_{k}$.

\section{CONSTRAINED DYNAMICS}

The Lie group variational dynamics for a controlled rigid spacecraft on $\mathrm{SO}(3) \times \mathrm{SO}(3)$ in discrete-time are given by [22], [13],

$$
\begin{aligned}
\left(h \Pi_{k}\right)^{\times} & =\mathbf{F}_{k} \mathbf{J}-\mathbf{J F}_{k}^{\mathrm{T}}, \\
\mathbf{C}_{k+1} & =\mathbf{C}_{k} \mathbf{F}_{k}, \\
\Pi_{k+1} & =\mathbf{F}_{k}^{\mathrm{T}} \Pi_{k}+h \mathbf{T}_{k},
\end{aligned}
$$

where $\Pi_{k} \in \mathbb{R}^{3}$ is the angular momentum, $\mathbf{F}_{k} \in \mathrm{SO}(3)$ is a one time-step change in $\mathbf{C}_{k} \in \mathrm{SO}(3)$, which is the spacecraft rotation, $\mathrm{T}_{k} \in \mathbb{R}^{3}$ is the applied torque, $h$ is the discretization time-step, and $\mathbf{J}=\frac{1}{2} \operatorname{tr}\left(\mathbf{J}_{c}\right) \mathbf{I}-\mathbf{J}_{c}$, where $\mathbf{J}_{c}$ is the spacecraft inertia matrix. For subsequent ease of exposition, we define a new variable, $\mathbf{X}_{k}=\left(\mathbf{C}_{k}, \mathbf{F}_{k}\right) \in \mathrm{SO}(3) \times \mathrm{SO}(3)$.

The dynamics are subject to the state- and controlconstraint,

$$
\left(\mathbf{X}_{k}, \mathbf{T}_{k}\right) \in \mathcal{C}, \forall k \in \mathbb{Z}_{+}
$$

where $\mathcal{C} \subset \mathrm{SO}(3) \times \mathrm{SO}(3) \times \mathbb{R}^{3}$ is a compact set with nonempty interior.

The solution to (1) proceeds by first computing $\mathbf{F}_{k}$ in (1a) from a given $\Pi_{k}$, which was computed by (1c) at the previous time-instant. This can be done through the solution of a continuous-time algebraic Riccati equation [23]. However, a special orthogonal solution $\mathbf{F}_{k}$ to (1a) exists if and only if [23],

$$
\left(h \Pi_{k}^{\times}\right)^{2}+4 \mathbf{J}^{2} \succeq 0 .
$$

This implies that, in order to guarantee a solution to (1a) at the next time step, the convex ${ }^{1}$ condition (3) must be enforced at the current time-step, i.e.,

$$
\left(h \Pi_{k+1}^{\times}\right)^{2}+4 \mathbf{J}^{2} \succeq 0 .
$$

Therefore, in addition to all other constraints, (4) is always included in the constraint set $\mathcal{C}$.

Remark 1: Condition (3) ensures the angular velocity $\Omega_{k}=\mathbf{J}_{c}^{-1} \Pi_{k}$ is small enough so there exists a solution $\mathbf{F}_{k}$ corresponding to $\Omega_{k}$. Physically, the change in rotation $\mathbf{F}_{k}$ can correspond to an infinite number of values of $\Omega_{k}$, but the solution to (1a) only corresponds to one.

The two methods that we present in the following sections utilize the above equations to propagate the given rotation and angular momentum in order to predict the constraint violation of the spacecraft and to ensure the tracking of a desired reference rotation.

\section{REFERENCE GOVERNOR}

Reference governors are predictive control schemes that modify a reference command to a closed-loop control system in order to enforce pointwise-in-time state and control constraints. They are applied to closed-loop systems that are designed to track the reference, usually without considering constraints. Reference governors use a state estimate to form a prediction and modify the reference in order to avoid constraint violation. A schematic is shown in Fig. 1.

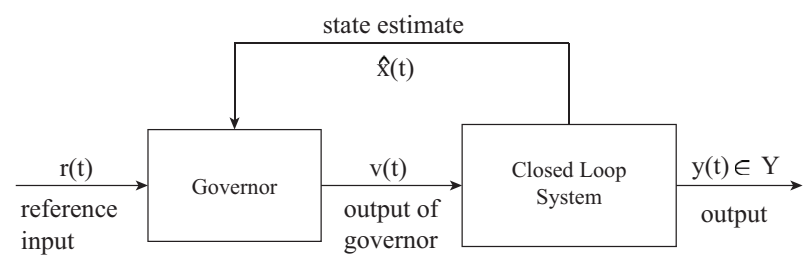

Fig. 1. Reference governor schematic

The reference governor described in [1], [2] is applied to an asymptotically stable closed-loop nonlinear system, which consists of an open-loop plant and a stabilizing controller with the reference as an input. The dynamics of this system are of the form,

$$
x_{k+1}=f\left(x_{k}, v_{k}\right),
$$

where $x_{k} \in \mathbb{R}^{n}$ is the state variable and $v_{k} \in \mathbb{R}^{m}$ is a reference input. Given a desired reference input $r_{k} \in \mathbb{R}^{m}$, the reference governor computes,

$$
v_{k}=\alpha_{k}\left(r_{k}-v_{k-1}\right)+v_{k-1},
$$

$$
\begin{gathered}
{ }^{1} x^{\mathrm{T}}\left(\left(h \Pi_{1}^{\times}\right)^{2}+4 \mathbf{J}^{2}\right) x \geq 0, x^{\mathrm{T}}\left(\left(h \Pi_{2}^{\times}\right)^{2}+4 \mathbf{J}^{2}\right) x \geq 0 \Longrightarrow \\
x^{\mathrm{T}}\left(\left(h\left(\left((1-\lambda) \Pi_{1}+\lambda \Pi_{2}\right)^{\times}\right)^{2}+4 \mathbf{J}^{2}\right) x=(1-\lambda)^{2} x^{\mathrm{T}}\left(\left(h \Pi_{1}^{\times}\right)^{2}+\right.\right. \\
\left.4 \mathbf{J}^{2}\right) x+\lambda^{2} x^{\mathrm{T}}\left(\left(h \Pi_{2}^{\times}\right)^{2}+4 \mathbf{J}^{2}\right) x+\lambda(1-\lambda) x^{\mathrm{T}}\left(h^{2} \Pi_{1}^{\times} \Pi_{2}^{\times}+h^{2} \Pi_{2}^{\times} \Pi_{1}^{\times}\right) x+ \\
2 \lambda(1-\lambda) x^{\mathrm{T}}\left(4 \mathbf{J}^{2}\right) x \geq(1-\lambda)^{2} x^{\mathrm{T}}\left(\left(h \Pi_{1}^{\times}\right)^{2}+4 \mathbf{J}^{2}\right) x+\lambda^{2} x^{\mathrm{T}}\left(\left(h \Pi_{2}^{\times}\right)^{2}+\right. \\
\left.4 \mathbf{J}^{2}\right) x+\lambda(1-\lambda) x^{\mathrm{T}}\left(\left(h \Pi_{1}^{\times}\right)^{2}+\left(h \Pi_{2}^{\times}\right)^{2}\right) x+2 \lambda(1-\lambda) x^{\mathrm{T}}\left(4 \mathbf{J}^{2}\right) x= \\
(1-\lambda) x^{\mathrm{T}}\left(\left(h \Pi_{1}^{\times}\right)^{2}+4 \mathbf{J}^{2}\right) x+\lambda x^{\mathrm{T}}\left(\left(h \Pi_{2}^{\times}\right)^{2}+4 \mathbf{J}^{2}\right) x \geq 0, \quad \forall \lambda \in[0,1]
\end{gathered}
$$


where $\alpha_{k} \in[0,1]$ is maximized subject to constraints being satisfied for all $t \in \mathbb{Z}_{+}$by the predicted response with $v_{k+t \mid k} \equiv v_{k}$ held constant. It is shown in [1], [2], that there exists a time $T^{*}$ such that if the constraints are satisfied for all $t \in \mathbb{Z}_{T^{*}}$, then they are satisfied for all $t \geq T^{*}$ if,

$$
v_{k} \in \mathcal{V}
$$

where $\mathcal{V}$ is a compact, nonempty, and convex set whose corresponding set of equilibrium points is contained in the interior of the set of all constraint-admissible equilibria.

\section{A. Unconstrained closed-loop control law}

The reference governor is a discrete-time control scheme that ensures pointwise-in-time constraint enforcement. Because it is applied to closed-loop systems, we need to develop a stabilizing nominal controller for the dynamics of the discrete-time model described in Section II. For this controller, we use the almost-globally stabilizing continuoustime control law, which, in the unconstrained case, guarantees $\mathbf{C}_{k} \rightarrow \mathbf{V}_{k} \in \mathrm{SO}(3)$ for almost every rotation $\mathbf{C}_{k}$. The closed-loop control design is described in the subsequent paragraph.

At every time-step $k$, given a constraint admissible reference rotation $\mathbf{V}_{k}$, we calculate the attitude error, which evolves on $\mathrm{SO}(3)$,

$$
\mathbf{E}_{k}=\mathbf{V}_{k}^{\mathrm{T}} \mathbf{C}_{k}
$$

We apply the feedback,

$$
\mathrm{T}_{k}=-\mathbf{K} \Pi_{k}-\mathrm{E}_{k},
$$

where $\mathbf{K} \in \mathbb{R}^{3 \times 3}$ is a positive-definite feedback gain and $\mathrm{E}_{k}$ is given by,

$$
\mathrm{E}_{k}^{\times}=\mathbf{A} \mathbf{E}_{k}-\mathbf{E}_{k}^{\mathrm{T}} \mathbf{A}
$$

for some symmetric positive-definite matrix $\mathbf{A} \in \mathbb{R}^{3 \times 3}$, for which no pair of eigenvalues is equal. In continuous-time, (9) can be shown to be asymptotically stabilizing [24] on all of $\mathrm{SO}(3) \times \mathbb{R}^{3}$ except for a set of measure zero, with stable equilibrium $\mathbf{E}_{k}=\mathbf{I}$ and unstable equilibria given by the set $\mathcal{U}_{\mathbf{A}}=\left\{\mathbf{E}_{k} \in \mathrm{SO}(3): \mathrm{E}_{k}^{\times}=0, \mathbf{E}_{k} \neq \mathbf{I}\right\}$; the set on which the control is not stabilizing is the union of all stable manifolds for the unstable equilibria in $\mathcal{U}_{\mathbf{A}}$. In this work, we assume that the continuous-time result is preserved in the discrete-time case for sufficiently small time-step $h$. Note that when $\mathbf{A}$ is diagonal, then $\mathcal{U}_{\mathbf{A}}=\left\{\mathbf{C}_{i}(\pi): i=1,2,3\right\}$, where $\mathbf{C}_{i}$ is an Euler rotation about the $i$-th axis from $\mathbf{I}$.

\section{B. Determining $\mathbf{V}_{k}$}

Because $\mathrm{SO}(3)$ is not closed under addition, the reference update equation (6) is not appropriate for references that are elements of the group. $\mathrm{SO}(3)$ is closed under multiplication however, so we introduce a new update equation similar to (6), but based on multiplication and exponentiation instead of addition and multiplication,

$$
\mathbf{V}_{k}=\left(\mathbf{R}_{k} \mathbf{V}_{k-1}^{\mathrm{T}}\right)^{\alpha_{k}} \mathbf{V}_{k-1} \in \mathcal{V}
$$

where $\mathcal{V} \subset \mathrm{SO}(3)$ is the constraint set for the reference, which satisfies the convexity-like condition,

$$
\mathbf{V}, \mathbf{V}^{\prime} \in \mathcal{V} \Longrightarrow\left(\mathbf{V}^{\prime} \mathbf{V}^{\mathrm{T}}\right)^{\alpha} \mathbf{V} \in \mathcal{V}, \forall \alpha \in[0,1]
$$

Note that the curve described by varying $\alpha_{k} \in[0,1]$ in (11) is the shortest geodesic connecting $\mathbf{V}_{k-1}$ and $\mathbf{R}_{k}$ [25]; also note that $\mathbf{V}_{k}=\mathbf{V}_{k-1}$ if $\alpha_{k}=0$ and $\mathbf{V}_{k}=\mathbf{R}_{k}$ if $\alpha_{k}=1$.

In order to avoid the unstable dynamics associated with the union of stable manifolds of $\mathcal{U}_{\mathrm{A}}$, we impose,

$$
\left(\mathbf{X}_{k}, \mathbf{V}_{k}\right) \in \mathcal{W}
$$

where $\mathcal{W}$ is positively invariant under the dynamics (1)-(2), (8)-(10), and $\mathbf{V}_{k}$ held constant over the prediction horizon. The set $\mathcal{W}$ can be chosen so as to be a sublevel set of the closed-loop Lyapunov function ${ }^{2}$ [26].

Thus at every time-step $k, \alpha_{k}$ is obtained through numerical optimization by choosing the largest value of $\alpha_{k}$ for which constraint admissibility can be guaranteed if the reference is kept constant for all future time-steps. In this way, we obtain a recursively feasible reference that guarantees constraint admissibility for all future time-steps. Specifically, we perform the following optimization online,

$$
\begin{aligned}
& \max \left\{\alpha_{k} \in[0,1]: \mathbf{V}_{k+t \mid k} \equiv \mathbf{V}_{k},\right. \\
& \text { (1)-(2), (8)-(10), (13) are satisfied for all } \left.t \in \mathbb{Z}_{T^{s}}\right\} .
\end{aligned}
$$

The optimization is performed through a bisection algorithm similar to [1], [2]. Note that the simulation is only performed until the time-instant, $T^{s}-1$, and this may not predict constraint violation for all future time. However, under the assumption that $T^{s} \geq T^{*}$ (see the beginning of the section), if all constraints are satisfied for all $t \in \mathbb{Z}_{T^{s}}$, they will also be satisfied for any $t \geq T^{s}$.

Due to the properties of $\mathrm{SO}(3)$, for some pair $\mathbf{R}_{k}$ and $\mathbf{V}_{k-1}$, (11) may not have a unique solution. This occurs when $\mathbf{R}_{k}$ is a cut point of $\mathbf{V}_{k-1}$, i.e. $\left(\mathbf{R}_{k} \mathbf{V}_{k-1}^{\mathrm{T}}\right)^{2}=\mathbf{I}$ but $\mathbf{R}_{k} \mathbf{V}_{k-1}^{\mathrm{T}} \neq \mathbf{I}$. In this case, both geodesics connecting $\mathbf{V}_{k-1}$ and $\mathbf{R}_{k}$ are equal in length and $\mathbf{R}_{k} \mathbf{V}_{k-1}^{\mathrm{T}}=\log \pi \mathbf{N}^{\times}$, for some $\mathrm{N} \in \mathbb{R}^{3}$ where $\|\mathrm{N}\|_{2}=1 .^{3}$

By the defintion of $\mathcal{V}$, both geodesics are contained in $\mathcal{V}$, so the choice of geodesic is arbitrary. Accordingly, our approach is to modify the target reference by,

$$
\mathbf{R}_{k}:=\exp \left(-\varepsilon \pi \mathrm{N}^{\times}\right) \mathbf{R}_{k}
$$

for some small $0<\varepsilon<1$, before performing the optimization (14). With this small modification of the reference, we guarantee a unique solution for the algorithm in (14).

The online algorithm to calculate $\alpha_{k}$ is now given. In the algorithm, the initial state values are set at the current state estimates and $\alpha_{\text {cand }}$, a candidate $\alpha_{k}$, is chosen. Simulations

\footnotetext{
${ }^{2}$ For example, let $\mathcal{W}=\{(\mathbf{X}, \mathbf{V}): V(\mathbf{X}, \mathbf{V}) \leq c\}$, where $V(\mathbf{X}, \mathbf{V})$ is a Lyapunov function for the continuous-time analog to (1)-(2), (8)-(10) and $c<\inf _{\mathbf{X} \in \mathcal{U}_{\mathbf{A}}} V(\mathbf{X}, \mathbf{I})$.

${ }^{3}$ In this paper, the map $\log : \mathrm{SO}(3) \rightarrow \mathfrak{s o}(3)$ is defined as in [25] with an extension to the case when $\operatorname{tr}(\mathbf{C})=-1 . \log (\mathbf{C})=\frac{\theta}{2 \sin \theta}(\mathbf{C}-$ $\mathbf{C}^{\mathrm{T}}$ ) if $0<|\theta|<\pi$, where $\operatorname{tr}(\mathbf{C})=1+2 \cos \theta$; if $\operatorname{tr}(\mathbf{C})=3$, then $\log (\mathbf{C})=0$, and if $\operatorname{tr}(\mathbf{C})=-1$, then $\log (\mathbf{C})=\pi \mathbf{N}^{\times}$.
} 
are then performed over a finite time horizon to determine if a constraint is violated. When $\alpha_{\text {cand }}$ converges to a preset tolerance, the algorithm stops.

Algorithm 1:

1) If $\left(\mathbf{R}_{k} \mathbf{V}_{k-1}^{\mathrm{T}}\right)^{2}=\mathbf{I}$ and $\mathbf{R}_{k} \mathbf{V}_{k-1}^{\mathrm{T}} \neq \mathbf{I}$, perform (15).

2) Initialize $\alpha_{\text {cand }}:=1$ and check constraint satisfaction for all times $t \in \mathbb{Z}_{T^{s}}$. If constraints are satisfied, set $\alpha_{k}:=1$ and exit.

3) Initialize $\alpha_{\max }:=1$ and $\alpha_{\min }:=0$.

4) Set $\alpha_{\text {cand }}:=\alpha_{\min }+\frac{1}{2}\left(\alpha_{\max }-\alpha_{\min }\right)$ and check constraint satisfaction for all times $t \in \mathbb{Z}_{T^{s}}$.

a) If constraints are satisfied, set $\alpha_{\min }:=\alpha_{\text {cand }}$.

b) Otherwise, set $\alpha_{\max }:=\alpha_{\text {cand }}$.

5) If $\alpha_{\max }-\alpha_{\min } \approx 0$ to a predetermined tolerance, set $\alpha_{k}:=\alpha_{\min }$ and exit.

6) Go to Step 4.

Using the above algorithm, we can state that under the assumption that $T^{s} \geq T^{*}$, the reference governor exhibits the properties of recursive feasibility and finite settling time.

Proposition 1: Assume that (i) $\mathbf{R}_{k} \equiv \mathbf{R}$ is constant for $k \in \mathbb{Z}_{+}$, (ii) $\mathbf{V}_{-1}$ is feasible for (14), and (iii) $T^{s} \geq T^{*}$.

Then the following holds: (i) the control scheme described by Algorithm 1 is recursively feasible, i.e. the solution $\mathbf{V}_{k}=$ $\mathbf{V}_{k-1}$ is always feasible for (14); (ii) the scheme ensures finite-time convergence to a constraint-admissible reference, i.e. there exists a $T^{c} \in \mathbb{Z}_{+}$such that $\mathbf{V}_{k}=\tilde{\mathbf{R}} \in \mathcal{V}$ for all $k \geq T^{c}$; (iii) and if $\mathbf{R} \in \mathcal{V}$, then $\tilde{\mathbf{R}}=\mathbf{R}$.

Proof: The proof follows from the theorems and propositions in [1], [2], with a modification needed due to Step 1 of Algortihm 1 to show that we are always able to avoid antipodal points by redefining the admissible and desired references according to the algorithm.

Specifically, if $\mathbf{R V}_{k-1}^{\mathrm{T}}=\exp \left(\pi \mathbf{N}^{\times}\right)$, then before modification, $\exp \left(-\varepsilon \pi \mathbf{N}^{\times}\right) \mathbf{R V}_{k-1}^{\mathrm{T}}=\exp \left(-\varepsilon \pi \mathbf{N}^{\times}\right) \exp \left(\pi \mathrm{N}^{\times}\right)=$ $\exp \left((1-\varepsilon) \pi \mathrm{N}^{\times}\right) \neq \exp \left(\pi \mathrm{N}^{\times}\right)$. Therefore, if $\alpha_{k} \neq 0$ for some $k \in \mathbb{Z}_{+}$, then $\mathbf{R V}_{k^{\prime}-1}^{\mathrm{T}} \neq \exp \left(\pi \mathrm{N}^{\times}\right)$for all $k^{\prime} \geq k$, if not, then $\tilde{\mathbf{R}}=\mathbf{V}_{k-1}$.

\section{MOdel PREDictive CONTROL ON SO(3)}

In this section, we propose a model predictive controller on $\mathrm{SO}(3) \times \mathrm{SO}(3)$ for spacecraft dynamics. This predictive control scheme solves a state- and control-constrained optimization problem over a finite horizon. Recursive feasibility and asymptotic stability of the resulting control sequence is guaranteed by an additional constraint that the state at the final time is contained in a terminal set, wherein a locally stabilizing controller satisfies the constraints in addition to a cost-decrease condition.

In order to derive the terminal set, we assume that the equilibrium $(\mathbf{I}, \mathbf{I}, 0) \in \operatorname{int} \mathcal{C}$, and linearize the rotational dynamics in order to obtain a locally stabilizing control.

\section{A. Linearization}

We linearize (1) around $\mathbf{X}_{k}=(\mathbf{I}, \mathbf{I})$ and $\mathrm{T}_{k}=0$ to obtain [13],

$$
\left[\begin{array}{l}
\delta \mathrm{Z}_{i+1} \\
\delta \Pi_{i+1}
\end{array}\right]=A\left[\begin{array}{l}
\delta \mathrm{Z}_{i} \\
\delta \Pi_{i}
\end{array}\right]+B \mathrm{~T}_{i} .
$$

where,

$$
A=\left[\begin{array}{cc}
\mathbf{I} & h \mathbf{J}_{c}^{-1} \\
0 & \mathbf{I}
\end{array}\right], B=\left[\begin{array}{c}
0 \\
h \mathbf{I}
\end{array}\right],
$$

and $\delta \mathrm{Z}_{i}$ and $\delta \Pi_{i}$ are linear approximations, for which $\delta \mathrm{Z}_{i}^{\times} \approx$ $\mathbf{Z}_{i}^{\times}=\log \mathbf{C}_{k}$ and $\delta \Pi_{i} \approx \Pi_{i}$ in a neighborhood of $(\mathbf{I}, \mathbf{I})$.

We also note that if $\left(\mathbf{C}_{i}, \mathbf{F}_{i}\right) \approx(\mathbf{I}, \mathbf{I})$, then as a linear approximation,

$$
\begin{aligned}
\operatorname{tr}\left(\mathbf{Q}_{1}\left(\mathbf{I}-\mathbf{C}_{i}\right)\right) & =\frac{1}{2}\left\|\mathbf{Q}_{1}^{1 / 2}\left(\mathbf{I}-\mathbf{C}_{i}\right)\right\|_{F}^{2} \\
& \approx \frac{1}{2}\left\|\mathbf{Q}_{1}^{1 / 2} \delta \mathbf{Z}_{i}^{\times}\right\|_{F}^{2} \\
& =\frac{1}{2} \delta \mathbf{Z}_{i}^{\mathrm{T}} \tilde{\mathbf{Q}}_{1} \delta \mathbf{Z}_{i}
\end{aligned}
$$

and similarly,

$$
\begin{aligned}
\frac{1}{h^{2}} \operatorname{tr}\left(\mathbf{Q}_{2}\left(\mathbf{I}-\mathbf{F}_{i}\right)\right) & =\frac{1}{2 h^{2}}\left\|\mathbf{Q}_{2}^{1 / 2}\left(\mathbf{I}-\mathbf{F}_{i}\right)\right\|_{F}^{2} \\
& \approx \frac{1}{2 h^{2}}\left\|\mathbf{Q}_{2}^{1 / 2} h\left(\mathbf{J}_{c}^{-1} \delta \Pi_{i}\right)^{\times}\right\|_{F}^{2} \\
& =\frac{1}{2} \delta \Pi_{i}^{\mathrm{T}} \mathbf{J}_{c}^{-1} \tilde{\mathbf{Q}}_{2} \mathbf{J}_{c}^{-1} \delta \Pi_{i},
\end{aligned}
$$

where $\tilde{\mathbf{Q}}_{1,2}=\operatorname{tr}\left(\mathbf{Q}_{1,2}\right) \mathbf{I}-\mathbf{Q}_{1,2}$ for symmetric positive definite $\mathbf{Q}_{1,2} \in \mathbb{R}^{3 \times 3}$.

\section{B. Model predictive controller}

Consider the cost function,

$$
\sum_{i=0}^{N-1} \lambda L\left(\mathbf{X}_{k+i \mid k}, \mathrm{~T}_{k+i \mid k}\right)+F\left(\mathbf{X}_{k+N \mid k}\right),
$$

where $0<\lambda<1$,

$$
\begin{aligned}
& L\left(\mathbf{X}_{i}, \mathbf{T}_{i}\right)= \\
& \operatorname{tr}\left(\mathbf{Q}_{1}\left(\mathbf{I}-\mathbf{C}_{i}\right)\right)+\frac{1}{h^{2}} \operatorname{tr}\left(\mathbf{Q}_{2}\left(\mathbf{I}-\mathbf{F}_{i}\right)\right)+\frac{1}{2}\left\|\mathbf{T}_{i}\right\|_{\mathbf{M}}^{2},
\end{aligned}
$$

for some symmetric positive-definite $\mathbf{M} \in \mathbb{R}^{3 \times 3}$, and,

$$
F\left(\mathbf{X}_{N}\right)=\frac{1}{2}\left\|\left(\mathrm{Z}_{N}, \Pi_{N}\right)\right\|_{\mathbf{P}}^{2}
$$

where $\mathbf{P}$ is the solution to the discrete-time algebraic Riccati equation,

$$
\mathbf{P}=A^{\mathrm{T}} \mathbf{P} A-\left(A^{\mathrm{T}} \mathbf{P} B\right)\left(\mathbf{M}+B^{\mathrm{T}} \mathbf{P} B\right)^{-1}\left(B^{\mathrm{T}} \mathbf{P} A\right)+\tilde{\mathbf{Q}},
$$

for $\tilde{\mathbf{Q}}=\operatorname{diag}\left(\tilde{\mathbf{Q}}_{1}, \mathbf{J}_{c}^{-1} \tilde{\mathbf{Q}}_{2} \mathbf{J}_{c}^{-1}\right)$.

Let $K=-\left(\mathbf{M}+B^{\mathrm{T}} \mathbf{P} B\right)^{-1}\left(B^{\mathrm{T}} \mathbf{P} A\right)$ and define,

$$
\kappa\left(\mathbf{X}_{i}\right)=K\left[\begin{array}{l}
\mathrm{Z}_{i} \\
\Pi_{i}
\end{array}\right]
$$

so that $\mathbf{T}_{i}=\kappa\left(\mathbf{X}_{i}\right)$ is an asymptotically stabilizing controller in a neighborhood of $(\mathbf{I}, \mathbf{I})$. Let $\mathcal{X}_{T} \subset \mathrm{SO}(3) \times \mathrm{SO}(3)$ be positively invariant with respect to the closed-loop dynamics (1) with control $\mathrm{T}_{k}=\kappa\left(\mathbf{X}_{k}\right)$ and satisfy the inclusion $\mathcal{X}_{T} \subset$ $\mathcal{P}$ where,

$$
\mathcal{P} \stackrel{\text { def }}{=}\left\{\mathbf{X}_{i}:\left\|\left(\mathbf{Z}_{i}, \Pi_{i}\right)\right\|_{\mathbf{P}}^{2} \leq c,\left(\mathbf{X}_{i}, \kappa\left(\mathbf{X}_{i}\right)\right) \in \mathcal{C}\right\} .
$$

${ }^{4}$ To see this, consider a symmetric positive definite $\mathbf{Q} \in \mathbb{R}^{3 \times 3}$. Then for every $\omega^{\times}=\sum_{i=1}^{3} \lambda_{i} j_{i}, \operatorname{tr}\left(\lambda_{i} j_{i} \mathbf{Q} \lambda_{i} j_{i}^{\mathrm{T}}\right)=\lambda_{i} \lambda_{i}\left(\operatorname{tr}(\mathbf{Q})-\mathbf{Q}_{i i}\right)$, and if $i \neq k$, then $\operatorname{tr}\left(\lambda_{i} j_{i} \mathbf{Q} \lambda_{k} j_{k}^{\mathrm{T}}\right)=-\lambda_{i} \lambda_{k} \mathbf{Q}_{i k}$. 
Note that in (29), $\left(\mathrm{Z}_{i}, \Pi_{i}\right)$ correspond to $\mathbf{X}_{i}$ as per their definition. The parameter $c>0$ is numerically determined to guarantee,

$$
F\left(\mathbf{X}_{i+1}\right)-F\left(\mathbf{X}_{i}\right) \leq-\lambda L\left(\mathbf{X}_{i}, \kappa\left(\mathbf{X}_{i}\right)\right)
$$

for some choice of the parameter $\lambda$. Given $0<\lambda<1$, consider the following problem,

$$
\max _{\mathbf{X}_{i}} F\left(\mathbf{X}_{i+1}\right)-F\left(\mathbf{X}_{i}\right)+\lambda L\left(\mathbf{X}_{i}, \kappa\left(\mathbf{X}_{i}\right)\right)
$$

subject to $\mathbf{X}_{i} \in \mathcal{P}$.

The parameter $c$ is chosen so that the solution to (31) is as close to zero as possible without being positive. Note that a positive solution always exists due to the fact that the accuracy of a linear approximation increases in smaller neighborhoods of the linearization. Specifically, because the system (16), (28) satisfies (30) for any $\lambda \leq 1$ and because $F$ and $L$ are continuous in their arguments, there exists a neighborhood of $(\mathbf{I}, \mathbf{I})$, in which nonlinear terms are bounded by $(1-\lambda) L\left(\mathbf{X}_{i}, \kappa\left(\mathbf{X}_{i}\right)\right)$.

The model predictive control is determined by solving the following optimization problem,

$$
\begin{aligned}
\underset{\left\{\mathrm{T}_{k+i \mid k}\right\}_{i=0}^{N-1}}{\min } & (24), \\
\text { subject to } & (1), \\
& \left(\mathbf{X}_{k+i \mid k}, \mathrm{~T}_{k+i \mid k}\right) \in \mathcal{C}, i \in \mathbb{Z}_{N}, \\
& \mathbf{X}_{k+N \mid k} \in \mathcal{X}_{T} .
\end{aligned}
$$

The control $\mathrm{T}_{k}=\mathrm{T}_{k \mid k}^{*}$ is set to the first element of the sequence solving (32).

Let the set of all initial conditions for which there exists a control input guiding the state to $\mathcal{X}_{T}$ in $N$ time-steps be defined as,

$$
\mathcal{R}_{N}=\left\{\mathbf{X}_{k}: \exists \mathrm{T}_{k+i \mid k}, i \in \mathbb{Z}_{N} \text { feasible for (32) }\right\} .
$$

Proposition 2: Let $\mathbf{X}_{0} \in \mathcal{R}_{N}$. Then the control law (32) asymptotically stabilizes $\mathbf{X}_{k}$ to the equilibrium $(\mathbf{I}, \mathbf{I})$.

Proof: The proof is based on using the value function of (32) as the Lyapunov function [3], [5]. Note that for all $(\mathbf{X}, \kappa(\mathbf{X})) \in \mathcal{C}, \lambda L(\mathbf{X}, \kappa(\mathbf{X})) \leq \lambda L(\mathbf{X}, 0)$ and $\lambda L(\mathbf{X}, 0)=$ $\left\|\mathbf{Q}_{1}^{1 / 2}(\mathbf{I}-\mathbf{C})\right\|_{F}^{2}+\left\|\mathbf{Q}_{2}^{1 / 2}(\mathbf{I}-\mathbf{F})\right\|_{F}^{2} \geq \alpha_{1}\left(\|\mathbf{I}-\mathbf{C}\|_{F}^{2}+\| I-\right.$ $\left.\mathbf{F} \|_{F}^{2}\right)$, where $\alpha_{1} \in \mathcal{K}_{\infty}$ and $\mathbf{X}=(\mathbf{C}, \mathbf{F})$.

Let $\left\{\mathrm{T}_{k \mid k}^{*}, \ldots, \mathrm{T}_{k+N-1 \mid k}^{*}\right\}$ be the optimal control of (32) and $\left\{\mathbf{X}_{k+1 \mid k}^{*}, \ldots, \mathbf{X}_{k+N \mid k}^{*}\right\}$ be the associated sequence of states. Then $\left\{\mathrm{T}_{k+1 \mid k}^{*}, \ldots, \mathrm{T}_{k+N-1 \mid k}^{*}, \kappa\left(\mathbf{X}_{k+N \mid k}^{*}\right)\right\}$ is feasible for (32) because of the positive invariance and constraint feasibility of $\mathcal{X}_{T}$.

Given state, $\mathbf{X}_{k}$, let $J^{*}\left(\mathbf{X}_{k}\right)$ be the minimum value in (32). Using (30), it can be shown that $J^{*}\left(\mathbf{X}_{k}\right)$ is a Lyapunov function because $J^{*}\left(\mathbf{X}_{k+1}\right)-J^{*}\left(\mathbf{X}_{k}\right) \leq-\lambda L\left(\mathbf{X}_{k}, \mathbf{T}_{k}\right) \leq$ $-\lambda L\left(\mathbf{X}_{k}, 0\right) \leq-\lambda \alpha_{1}\left(\|\mathbf{I}-\mathbf{C}\|_{F}^{2}+\|\mathbf{I}-\mathbf{F}\|_{F}^{2}\right)$.

The following proposition states that if there are no constraints on the spacecraft rotation $\mathbf{C}_{k}$ in steady state, then we are always able to stabilize an arbitrary inital rotation with a small initial angular velocity to the equilibrium.
Proposition 3: Assume $\operatorname{int} \mathcal{C} \supset \operatorname{SO}(3) \times\{\mathbf{I}\} \times\{0\}$. Then there exists $N^{*} \in \mathbb{Z}_{+}$and an open set $V \subset \mathrm{SO}(3)$ such that $\mathbf{I} \in V$ and $\mathcal{R}_{N^{*}} \supset \mathrm{SO}(3) \times V$.

Proof: The set $\mathcal{C}$ has nonempty interior, so there exist open sets $V \subset \mathrm{SO}(3)$ and $U \subset \mathbb{R}^{3}$ containing $\mathbf{I}$ and 0 , respectively, such that $\mathcal{C} \supset \mathrm{SO}(3) \times V \times U$ and such that if $\mathbf{F}_{k} \in V$, there exists $\mathrm{T}_{k} \in U$ so that at the next timeinstant, $\mathbf{F}_{k+1}=\mathbf{I}$, and if $\mathbf{F}_{k+1} \in V$, there exists $\mathrm{T}_{k} \in U$ so that at the current time-instant, $\mathbf{F}_{k}=\mathbf{I}$. Note that the latter condition can always be satisfied by choosing $V$ to be smaller.

Let $\mathcal{S}_{\mathbf{C}}=\{\mathbf{C F} \in \mathrm{SO}(3): \mathbf{F} \in V\}$ be the set of all rotations that are reachable from $\mathbf{C}$ with $\mathbf{F} \in V$. Therefore, by the application of two controls, $\mathrm{T}_{k}$ and $\mathrm{T}_{k+1}$, we are able to rotate $\mathbf{C}_{k}$ to any $\mathbf{C}_{k+2} \in \mathcal{S}_{\mathbf{C}_{k}}$.

Let $\mathcal{A}$ be the enumeration of all elements $\mathbf{C}^{i} \in \mathrm{SO}(3)$. Because $\mathcal{X}$ is compact and $\mathcal{S}_{\mathbf{C}^{i}} \times V$ are open in $\operatorname{SO}(3) \times$ $\mathrm{SO}(3)$, there exists a finite $F \subset \mathcal{A}$ such that $\cup_{i \in F} \mathcal{S}_{\mathbf{C}^{i}} \times V=$ $\cup_{i \in \mathcal{A}} S_{\mathbf{C}^{i}} \times V$. This implies that there exists a finite control sequence that guides the system to the terminal set from an arbitrary rotation that is close to rest.

Remark 2: Proposition 3 implies that if the continuoustime spacecraft dynamics on $\mathrm{SO}(3) \times \mathbb{R}^{3}$ are unconstrained, we are able to form a globally stabilizing discrete-time controller: If the spacecraft has large angular velocity $\Omega_{k}$, we can detumble the spacecraft by using a closed-loop feedback control, then when $\Omega_{k}$ is small enough so that $\mathbf{F}_{k} \in V$, the result of Proposition 3 shows that we can guide an arbitrary rotation to the equilibrium.

Corollary 4: If int $\mathcal{C} \supset \mathrm{SO}(3) \times \mathrm{SO}(3) \times\{0\}$, then there exists $N^{*} \in \mathbb{Z}_{+}$such that $\mathcal{R}_{N^{*}}=\mathrm{SO}(3) \times \mathrm{SO}(3)$.

\section{NUMERICAL RESUlts}

In this section, we consider a spacecraft with inertia matrix $\mathbf{J}_{c}=\operatorname{diag}(10,8,8)$ and discretization time-step $h=0.1$. In the figures, we plot the orientation maneuvers on the sphere $\mathrm{S}^{2}$, where the vector $\left[\begin{array}{lll}x & y & z\end{array}\right]^{\mathrm{T}}$, corresponding to the first column of $\mathbf{C}_{k}$ is plotted in green, the second is in blue, and the third is in red.

\section{A. Reference governor simulation}

For the reference governor, we consider two constraints: a pointing inclusion constraint and a thrust limit. The pointing inclusion constraint is given as a constraint that the spacecraft point towards a fixed target, such as the Earth; it can also be considered as an exclusion constraint, requiring the spacecraft not point outside of the inclusion zone, e.g. so as to not damage photosensitive equipment, we may require that the spacecraft not point towards the Sun. The inclusion constraint that we consider here is that the spacecraft point within $60^{\circ}$ of the fixed axis, $e_{3}$. This can be expressed as a constraint on the $(3,3)$ entry of the matrix, $\mathbf{C}_{k}$,

$$
\mathbf{C}_{k, 33} \geq \cos 60^{\circ}=0.5 \text {. }
$$

The other constraint is a limit on the thrust force, which is expressed as,

$$
\left\|\mathrm{T}_{k}\right\|_{2} \leq 0.02
$$




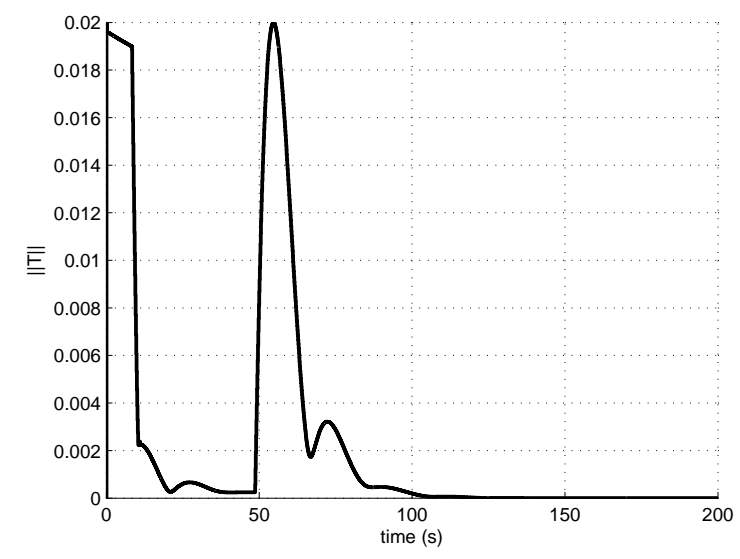

Fig. 2. $\left\|\mathrm{T}_{k}\right\|_{2}$ corresponding to the reference governor simulation

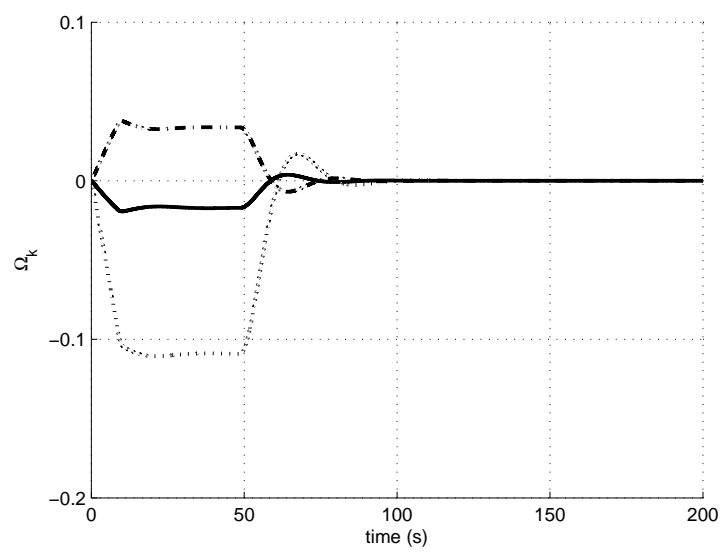

Fig. 3. $\Omega_{k, 1}$ (solid), $\Omega_{k, 2}$ (dot-dash), and $\Omega_{k, 3}$ (dotted) corresponding to the reference governor simulation

We choose the set $\mathcal{V}$ as a compact set, the elements of which strictly satisfy (33) in steady state.

Figs. 2-5 present simulation results corresponding to the reference governor simulation, with closed-loop control gains $\mathbf{A}=\operatorname{diag}(0.1,0.2,0.3)$ and $\mathbf{K}=0.2 \mathbf{I}$. The reference governor modifies the reference to command the rotation, while enforcing all constraints, from an initial condition close to the inclusion constraint boundary to the reference rotation $\mathbf{R}_{k} \equiv \mathbf{I}$. The only constraint that becomes active in the closed-loop trajectory is the control constraint, which is plotted in Fig. 2; the corresponding time history of the reference governor parameter $\alpha_{k}$, is plotted in Fig. 4 and shows that the reference governor modifies the desired rotation signal until the desired reference is admissible. In Fig. 5, we plot the orientation maneuver of the spacecraft along with the trajectory of the admissible reference $\mathbf{V}_{k}$.

\section{B. Model predictive control simulation}

In this section, we present two numerical examples utilizing the model predictive control method from Section IV. In the first example, the controller stabilizes an initial rotation from the initial condition of the preceding subsection to the

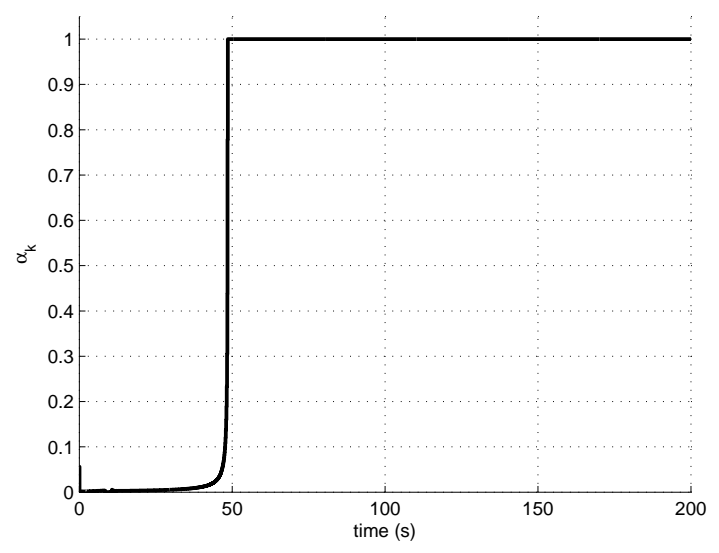

Fig. 4. $\alpha_{k}$ corresponding to the reference governor simulation

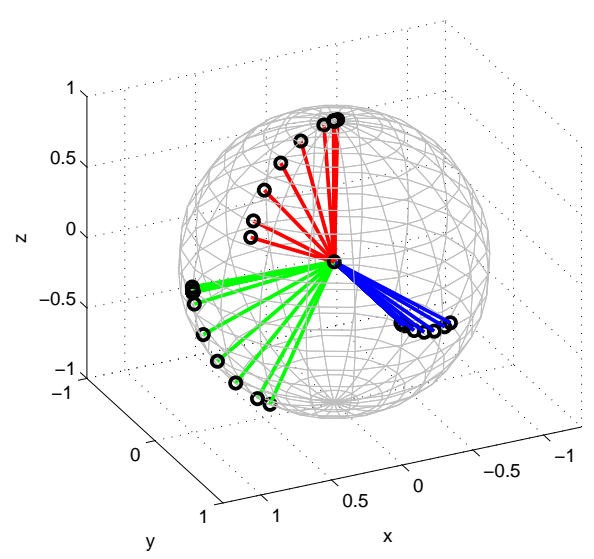

Fig. 5. Orientation maneuver corresponding to the reference governor simulation plotted at $10 \mathrm{~s}$ increments; the lines connecting the points on the sphere represent the path of the reference trajectory

equilibrium, $\mathbf{C}_{k}=\mathbf{I}$. In the second example, we simulate a rest-to-rest rotation from $\mathbf{C}_{3}(\pi)$ to $\mathbf{I}$.

The design of the controller uses weights, $\mathbf{Q}_{1}=\mathbf{I}, \mathbf{Q}_{2}=$ $\mathbf{J}, \mathbf{M}=1000 \mathbf{I}$, and $\lambda=0.1$. The terminal constraint set is chosen as $\mathcal{X}_{T}=\mathcal{P}$ in both cases.

In the first case, we let $N=5$ and consider only the control constraint (34) with the same initial condition as in the previous example. The results are presented in Figs. 6-8, where we show in Fig. 6 that the constraint is satisfied and in Fig. 8 that the target orientation is achieved. Comparing the results here with those of the previous section, we see that the rotation trajectories are similar.

In the second case, we let $N=10$ in order to expand the region of attraction. Our goal is to investigate the stabilizing properties of the controller. As a consequence of the Lefschetz-Hopf Theorem, all globally stabilizing continous time controllers must be discontinuous; we now check if this is true of our controller. To do this, we do not need to consider constraints on the control input, so we choose a large constraint set $\mathcal{C}$, ensuring that the constraints never become active; we also decrease the control cost to $\mathbf{M}=$ 


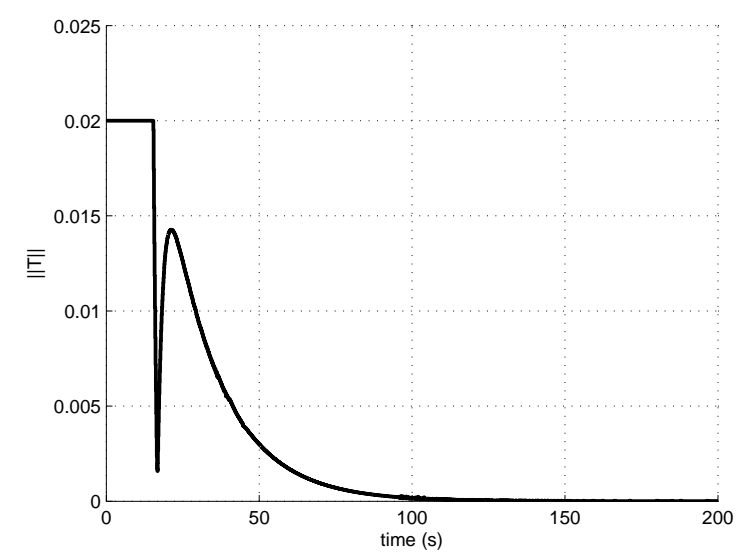

Fig. 6. $\left\|\mathrm{T}_{k}\right\|_{2}$ corresponding to the model predictive control simulation

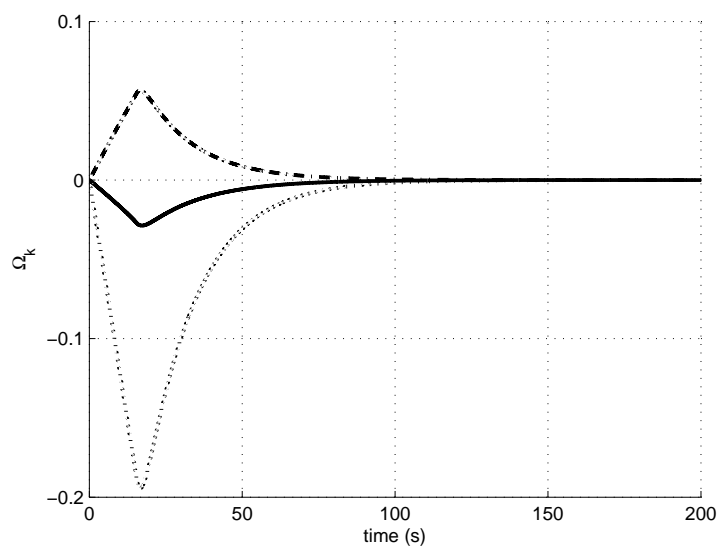

Fig. 7. $\Omega_{k, 1}$ (solid), $\Omega_{k, 2}$ (dot-dash), and $\Omega_{k, 3}$ (dotted) corresponding to the model predictive control simulation

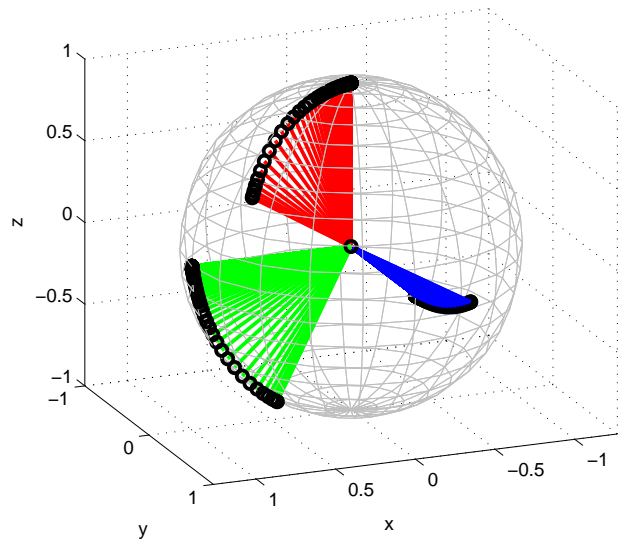

Fig. 8. Orientation maneuver corresponding to the model predictive control simulation plotted at $2 \mathrm{~s}$ increments

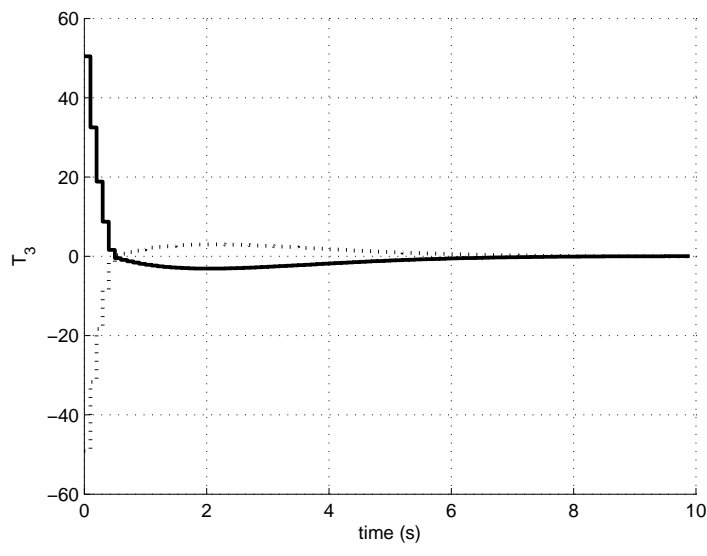

Fig. 9. $\mathbf{T}_{k, 3}$ for initial condition $\mathbf{C}_{3}(\pi)$ (solid) and $\mathbf{C}_{3}(-0.99 \pi)$ (dotted)

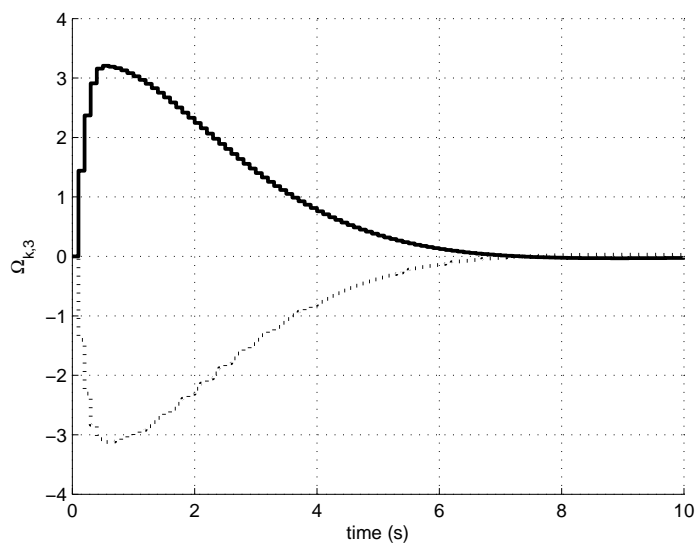

Fig. 10. $\Omega_{k, 3}$ for initial condition $\mathbf{C}_{3}(\pi)$ (solid) and $\mathbf{C}_{3}(-0.99 \pi)$ (dotted)

I in order to allow larger control inputs. The results for two simulations are presented in Figs. 9-12. In the first simulation, the initial rotation is $\mathbf{C}_{3}(\pi)$, and in the second case, it is $\mathbf{C}_{3}(-0.99 \pi)$. The torque and angular velocity on the $z$-axis are shown in Figs. 9-10, respectively, where we can see that, although the initial conditions are very close to each other, the trajectories are almost opposite in sign. This shows that there is a discontinuity in the control occurring near $\mathbf{C}_{3}(\pi)$. In fact, it occurs exactly at $\mathbf{C}_{3}(\pi)$ or at any resting initial condition, for which $\operatorname{tr}(\mathbf{C})=-1$; the discontinuity is a consequence of the restriction of the Log function range. For example, in our solution, we chose the range of $\log \mathbf{C}_{3}(\theta)$ to be $\left\{\theta j_{3}: \theta \in(-\pi, \pi]\right\}$; if we had instead restricted $\theta \in(-\pi / 2,3 \pi / 2]$, then the control for the cases considered above would not be discontinuous, but a discontinuity would nevertheless exist at the initial condition $\mathbf{C}_{3}(3 \pi / 2)$.

In Figs. 11-12, the direction of rotation is marked with an arrow. We see that in one case, the control rotates the spacecraft in the counter-clockwise direction, whereas in the other case, the rotation is clockwise. 


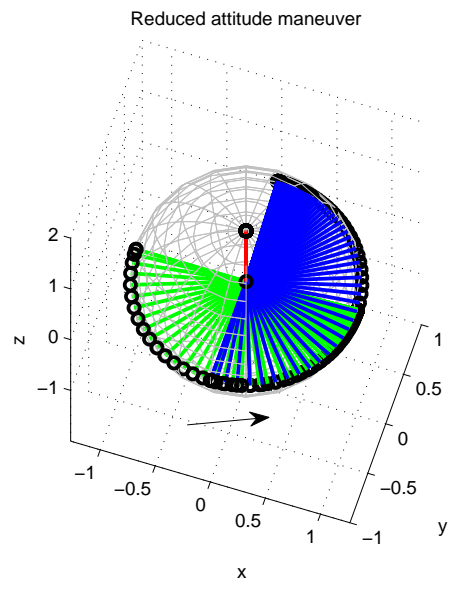

Fig. 11. Orientation maneuver corresponding to the initial condition $\mathbf{C}_{3}(\pi)$, plotted at $2 \mathrm{~s}$ increments

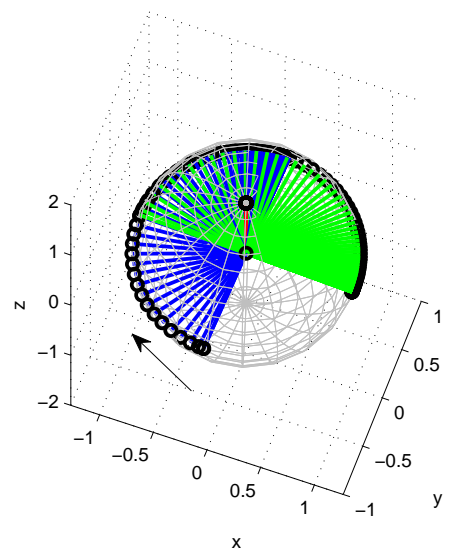

Fig. 12. Orientation maneuver corresponding to the initial condition $\mathbf{C}_{3}(-0.99 \pi)$, plotted at $2 \mathrm{~s}$ increments

\section{CONCLUSION}

In this paper, we considered the problem of constrained control of spacecraft dynamics and presented two predictive control schemes, a reference governor and a nonlinear model predictive controller. Both schemes used the Lie group variational integrator to evolve their predictions on $\mathrm{SO}(3) \times$ $\mathrm{SO}(3)$. We showed that both schemes guarantee constraint admissibility and convergence to the desired equilibrium and presented numerical results exhibiting these properties.

We also showed that in the unconstrained case, both schemes are globally stabilizing. Because globally stabilizing controllers are necessarily discontinuous, we also numerically demonstrated a discontinuity in the control.

\section{ACKNOWLEDGMENT}

The authors acknowledge Prof. N. H. McClamroch for providing helpful suggestions.

\section{REFERENCES}

[1] A. Bemporad, "Reference governor for constrained nonlinear systems," IEEE Trans. Automat. Control, vol. 43, no. 3, pp. 415-419, 1998.
[2] E. Gilbert and I. Kolmanovsky, "Nonlinear tracking control in the presence of state and control constraints: A generalized reference governor," Automatica, vol. 38, no. 12, pp. 2063-2073, 2002.

[3] D. Q. Mayne, J. B. Rawlings, C. V. Rao, and P. O. M. Scokaert, "Constrained model predictive control: Stability and optimality," $\mathrm{Au}$ tomatica, vol. 36, no. 6, pp. 789-814, 2000.

[4] E. F. Camacho and C. Bordons, Model Predictive Control, 2nd Ed., London, England: Springer, 2004.

[5] G. Goodwin, M. Seron, and J. A. De Doná, Constrained Control and Estimation: An Optimization Approach, London, England: Springer, 2005.

[6] L. Grüne and J. Pannek, Nonlinear Model Predictive Control. London, England: Springer, 2011.

[7] E. Silani and M. Lovera, "Magnetic spacecraft attitude control: a survey and some new results," Control Eng. Practice, vol. 13, no. 3, pp. 357-371, 2005.

[8] Ø. Hegrenæs, J. T. Gravdahl, and P. Tøndel, "Spacecraft attitude control using explicit model predictive control," Automatica, vol. 41, no. 12 , pp. 2107-2114, 2005.

[9] J. Vandersteen, M. Diehl, C. Aerts, and J. Swevers, "Spacecraft attitude estimation and sensor calibration using moving horizon estimation," J. Guidance Control Dynamics, vol. 36, no. 3, pp. 734-741, 2013.

[10] S. Gros, M. Zanon, M. Vukov, and M. Diehl, "Nonlinear MPC and MHE for mechanical multi-body systems with application to fast tehtered airplanes," in Proc. Nonlinear Model Predictive Control Conf., Noordwijkerhout, Netherlands, 2012, pp. 86-93.

[11] T. Lee, N. H. McClamroch, and M. Leok, "A Lie group variational integrator for the attitude dynamics of a rigid body with applications to the 3D pendulum," in Proc. IEEE Conf. Control Applicat., Toronto, ON, 2005, pp. 962-967.

[12] T. Lee, M. Leok, and N. H. McClamroch, "Lie group variational integrators for the full body problem in orbital mechanics," Celestial Mech. Dynamical Astronomy, vol. 98, no. 2, pp. 121-144, 2007.

[13] T. Lee, M. Leok, and N. H. McClamroch, "Optimal attitude control of a rigid body using geometrically exact computations on $\mathrm{SO}(3)$," J. Dynamical Control Syst., vol. 14, no. 4, pp. 465-487, 2008.

[14] T. Lee, "Computational geometric mechanics and control of rigid bodies," Ph. D. dissertation, Dept. Aerosp. Eng., Univ. Michigan, Ann Arbor, 2008.

[15] A. M. Bloch, I. I. Hussein, M. Leok, and A. K. Sanyal, "Geometric structure-preserving optimal control of the rigid body," J. Dynamical Control Syst., vol. 15, no. 3, pp. 307-330, 2009.

[16] N. Nordkvist and A. K. Sanyal, "Attitude feedback tracking with optimal attitude state estimation," in Proc. American Control Conf., Baltimore, MD, 2010, pp. 2861-2866.

[17] N. A. Chaturvedi, A. K. Sanyal, and N. H. McClamroch, "Rigid body attitude control: Using rotation matrices for continuous, singularityfree control laws," IEEE Control Syst. Mag., vol. 31, no. 3, pp. 30-51, 2011.

[18] J. E. Marsden and M. West, "Discrete mechanics and variational integrators," Acta Numerica, vol. 10, pp. 357-514, 2001.

[19] D. V. Zenkov, M. Leok, and A. M. Bloch, "Hamel's formalism and variational integrators on a sphere," in Proc. IEEE Conf. Decision and Control, Maui, HI, 2012, pp. 7504-7510.

[20] E. S. Meadows, M. A. Henson, J. W. Eaton, and J. B. Rawlings, "Receding horizon control and discontinuous state feedback stabilization," Int. J. Control, vol. 62, no. 5, pp. 1217-1229, 1995.

[21] V. Guillemin and A. Pollack, Differential Topology, Englewood Cliffs, NJ: Prentice Hall, 1974.

[22] J. Moser and A. Veselov, "Discrete versions of some classical integrable systems and factorization of matrix polynomials," Commun. Math. Physics, vol. 139, no. 2, pp. 217-243, 1991.

[23] J. R. Cardoso and F. S. Leite, "The Moser-Veselov equation," Linear Algebra Applicat., vol. 360, pp. 237-248, 2003.

[24] A. Sanyal, A. Forsbury, N. Chaturvedi, and D. S. Bernstein, "Inertiafree spacecraft attitude tracking with disturbance rejection and almost global stabilization," J. Guidance Control Dynamics, vol. 32, no. 4, pp. 1167-1178, 2009.

[25] M. Moakher, "Means and averaging in the group of rotations," SIAM J. Matrix Anal. Applicat., vol. 24, no. 1, pp. 1-16, 2002.

[26] T. Lee, "Geometric tracking control of the attitude dynamics of a rigid body on SO(3)," in Prof. American Control Conf., San Francisco, CA, 2011, pp. 1200-1205. 